\title{
7 Leistungssteuerung mit Kostensensiblen Leitlinien: rechtliche Implikationen
}

Stefan Huster und Christian Held

\subsection{Die Notwendigkeit und grundsätzliche Zulässigkeit von Prioritätensetzungen und Leistungsbeschränkungen}

\subsubsection{Die Begrenztheit der Ressourcen}

Kostensensible Leitlinien (KSLL) beruhen auf dem - anderen Gesundheitssystemen vertrauten - Gedanken, dass medizinische Leistungen in einem öffentlichen System der Gesundheitsversorgung nicht unabhängig von den Kosten beansprucht werden können, da ansonsten das gesamte Bruttosozialprodukt in die medizinische Versorgung investiert werden könnte. Bereits diese Einsicht in die notwendige Begrenztheit der Ressourcen legt gegenüber einer grundsätzlichen verfassungsrechtlichen Abwehr von Leistungseinschränkungen aus Kostengründen Zurückhaltung nahe. So hat auch das Bundesverfassungsgericht bereits ausdrücklich bestätigt, dass es verfassungsrechtlich zulässig ist, Leistungen nur nach Maßgabe eines allgemeinen, von finanzwirtschaftlichen Erwägungen mitbestimmten Leistungskatalogs zu erbringen. ${ }^{12}$ Schließlich sei die Gesetzliche Krankenversicherung (GKV) nicht verpflichtet, all das zu leisten, was überhaupt an Methoden zur Erhaltung oder Wieder-

12 Vgl. BVerfG, Amtliche Sammlung (BVerfGE) 115, 25 (46f.); BVerfG (Kammer), Beschluss vom 30.6.2008, 1 BvR 1665/07, Rn. 10 der Juris-Fassung. 
herstellung der Gesundheit verfügbar ist. ${ }^{13}$ Die Rechtsprechung hat daher dem Kostenaspekt im Gesundheitswesen für gesetzgeberische Entscheidungen erhebliches Gewicht zugesprochen. ${ }^{14}$

\subsection{2 „Rationalisierung vor Rationierung“ als verfassungsrechtliches Gebot?}

Allerdings sind die Voraussetzung der Mittelknappheit und die sich daraus ergebende Notwendigkeit der Leistungseinschränkung (,Rationierung“) nicht unumstritten. ${ }^{15}$ Vielmehr wird häufig auch die Ansicht vertreten, dass in der GKV noch Effizienzreserven existieren, die zunächst gehoben werden können, ohne Abstriche an der Versorgungsqualität zu machen (,Rationalisierung“).. ${ }^{16}$ Politisch ist es durchaus vernünftig und nachvollziehbar, sich an dem Grundsatz „Rationalisierung vor Rationierung“ zu orientieren: Solange Ineffizienzen, Fehlsteuerungen und enorme Profite der Pharmaindustrie zu beobachten sind, ist es tatsächlich nicht nahe liegend, die Kosten vorrangig über Leistungsbeschränkungen in den Griff zu bekommen. ${ }^{17}$

Aus einer (verfassungs-)rechtlichen Perspektive hat dies allerdings nur geringe Bedeutung: Zum einen werden noch vorhandene Einsparungsmöglichkeiten langfristig wohl nicht ausreichen, um den Ausgabenanstieg durch den medizinisch-technischen Fortschritt und die demographische Entwicklung auszugleichen, sodass es nur eine Frage der Zeit ist, wann Leistungseinschränkungen - auch im verfassungsrechtlichen Sinn des Wortes - erforderlich werden. ${ }^{18}$ Zum anderen ist unklar, welche Rationalisierungspotenziale in welchen Bereichen tatsächlich noch bestehen und wie sie gehoben werden können. ${ }^{19}$ Daher hat der Gesetzgeber insoweit einen großen Einschätzungsspielraum. Wenn er meint, dass auf Leistungseinschränkungen zur Kostenbegrenzung nicht verzichtet werden kann, ist das vertretbar. ${ }^{20}$ Das Verfassungsrecht wird insoweit keine grundsätzlichen Hürden errichten. Auch die Rechtsprechung

13 Vgl. BVerfG, Neue Juristische Wochenschrift 1997, 3085 (3085); Bundessozialgericht (BSG), Urteil vom 28.2.2008, B 1 KR 16/07 R, Rn. 46 der Juris-Fassung.

14 Vgl. BVerfGE 103, 172 (184); BSG, Urteil vom 25.6.2009, B 3 KR 3/08 R, Rn. 25 der Juris-Fassung.

15 Weit überwiegend jedoch werden Leistungseinschränkungen für unumgänglich gehalten, vgl. Dabrock 2005: 214; Höfling/Augsberg 2009: 46; Hoppe 2008: 304; Kingreen 2008: 148; Kopetsch 2000: 69, von Maydell 2005: 88f.; sowie bereits Ebsen 1997: 111; Krämer 1996: 1ff.; und Oberender 1998: 16. Von Politikern hingegen wird gerne nach wie vor vertreten, es gebe kein Rationierungsproblem, vgl. die Zitate bei Wagner 2009: 7.

16 Vgl. Augurzky et al. 2009, Glaeske 2007: 553ff.; Schröder/Nink 2007: 305ff.; Vogt 2004: 189.

17 Vgl. Heiß 2003: 128.

18 So auch Höfling 1998: 144; Kingreen 2008: 152; Kliemt 2006: 47; Kollwitz 1999: 20; Kuhlmann 1998: 11; Marckmann 2005: 189; von Maydell 2004: 354. Hingegen kritisch zur Annahme der Kostensteigerung durch den medizinisch-technischen Fortschritt und die demographische Entwicklung Bosbach/Bingler 2008: 5ff.; Braun/ Kühn/Reiners 1998: 21ff.; Deppe 1998: 104f.; Kühn 2005: 178ff.; Reiners 2009: 17ff.

19 Vgl. Marckmann 2008: 890.

20 Vgl. BVerfGE 96, 330 (340), 103, 172 (183f. und 189); BSGE 69, 76 (80); Jaeger 2003: 233; Krause 1982: 177; Schlegel 2004: 341. 
7.1 Die Notwendigkeit und grundsätzliche Zulässigkeit von Prioritätensetzungen und Leistungsbeschränkungen

hat die bereits erfolgten Leistungseinschränkungen bisher verfassungsrechtlich gebilligt. ${ }^{21}$

\subsubsection{Kostensensible Leitlinien im Kontext der Priorisierungs- und Rationierungskriterien}

Es sind viele und ganz unterschiedlich ansetzende Möglichkeiten der Priorisierung und Rationierung bei begrenzten Mitteln denkbar: von Wartelisten über Zuzahlungspflichten bis zu Altersgrenzen. ${ }^{22}$ Besondere Bedeutung werden aber vermutlich immer „medizinnahe“ Kriterien haben, die abstellen auf die medizinische Bedürftigkeit (verstanden als Dringlichkeit der Behandlung), den medizinischen Nutzen und die Kosteneffektivität einer Maßnahme. ${ }^{23}$

Intuitiv ist es tatsächlich nahe liegend, Maßnahmen zu priorisieren, die auf besonders schwere Krankheiten reagieren, die dabei besonders viel gesundheitlichen Nutzen stiften und dies zu einem akzeptablen Preis tun. Diese intuitive Plausibilität beruht nicht auf einem kruden Utilitarismus, sondern lässt sich rechtfertigen über das Interesse, das jeder einzelne von uns an einem effizienten Mitteleinsatz hat - weil dann die Chance am größten ist, dass auch er selbst in den Genuss eines möglichst großen medizinischen Nutzens kommt. ${ }^{24}$ Cenau darauf beruht nun auch die Attraktivität von KSLL, wenn sie insbesondere auf den letztgenannten Punkt, die Kosteneffektivität, abstellen und Maßnahmen mit einem besonders schlechten Kosten-Nutzen-Verhältnis posteriorisieren.

\subsubsection{Verbot der „Bewertung“ von Gesundheit und Leben?}

Allerdings machen KSLL diese Überlegung - ebenso wie Kosten-Nutzen-Bewertungen, auf denen sie beruhen - besonders deutlich: Ein medizinischer Zusatznutzen wird aus Kostengründen vorenthalten. Tatsächlich ist damit eine „Bewertung“ von Gesundheit und ggf. sogar Leben impliziert, indem ein bestimmter gesundheitlicher Zusatznutzen als „zu teuer“ eingeschätzt wird. Ist eine solche „Bewertung“ nicht von vornherein unzulässig? Es gibt durchaus Anhaltspunkte in der Rechtsprechung des Bundesverfassungsgerichts, dass jedenfalls in Bezug auf das Rechtsgut „Leben“ keine (monetäre) Bewertung stattfinden darf. ${ }^{25}$

21 Vgl. zuletzt - zum Ausschluss der nicht verschreibungspflichtigen Arzneimittel in $\$ 34$ Abs. 1 S. 1 SGB V - BVerfG NJW 2013, 1220ff.

22 Zu Altersgrenzen vgl. jetzt umfassend Held 2011.

23 Zu diesen Kriterien vgl. etwa Zentrale Ethikkommission bei der Bundesärztekammer (ZEKO) 2007: 22ff.

$24 \mathrm{Vgl}$. dazu Huster 2010: 1073ff.

25 Vgl. BVerfGE 39, 1 (59); BVerfGE 115 (151ff.). 
Dies ist in sich schon eine sehr problematische Rechtsprechung. Im hiesigen Kontext sind zudem zwei spezielle Aspekte zu berücksichtigen:

• Nähme man diese Forderung eines Bewertungsverbots ernst, wären Gesundheitsausgaben - abgesehen von Randbereichen mit marginaler gesundheitlicher Bedeutung - überhaupt nicht mehr begrenzbar. Dies kann angesichts des medizinisch-technischen Fortschritts und der demographischen Entwicklung kein zielführender Weg sein und wäre auch ein Rigorismus, der nicht nur die Opportunitätskosten der medizinischen Versorgung ignorierte, ${ }^{26}$ sondern auch zur Praxis in anderen Gesundheitssystemen in einem seltsamen Widerspruch stände.

- Ein Bewertungsverbot mag noch nachvollziehbar sein, wenn es um individuelles Leben und individuelle Gesundheit geht: Person A soll als solcher kein höherer oder geringerer Wert als Person B beigemessen werden. Aber nicht darum geht es bei KSLL, sondern um Allokationsentscheidungen auf höherer Ebene, die zunächst rein statistisch ansetzen und nicht individuelle Personen „bewerten“. Derartige Bewertungen nehmen wir in der Politik andauernd vor, innerhalb des Gesundheitswesens (wie viele Intensivbetten? wie viele Rettungshubschrauber?) und außerhalb (welche Sicherheitsvorkehrungen sind im Straßenverkehr oder im Umweltschutz für Gesundheit und Leben besonders dringlich, welche „bringen zu wenig“?). Das heißt nicht, dass sich aus dem Verfassungsrecht gar keine Vorgaben für kostenbezogene Leistungseinschränkungen ergeben; ${ }^{27}$ es kann aber nicht sein, dass ein Gemeinwesen gar nicht mehr darüber nachdenken darf, welche Ressourcen in die Gesundheitsversorgung investiert werden sollen. ${ }^{28}$

\subsubsection{Die Alternative der impliziten Rationierung und ihre rechtliche Problematik}

Dabei ist immer auch zu berücksichtigen, dass die mit hoher Wahrscheinlichkeit zu erwartende Alternative noch unerfreulicher ist: Scheut man offene Leistungsbeschränkungen, muss die Bewältigung der Mittelknappheit der alltäglichen Versorgungspraxis überlassen werden. Dass dies im deutschen Gesundheitssystem bereits geschieht, wird zunehmend beklagt ${ }^{29}$ und ist auch im Zusammenhang dieses Forschungsverbundes untersucht worden ${ }^{30}$. Eine derartige - etwa durch Budgetierungen und Kostendeckelungen verursachte sog. implizite Rationierung mag für die gesundheitspolitischen Entschei-

26 Zur normativen Bedeutung dieses Begriffs vgl. Huster/Kliemt 2009: 241ff.

27 Zu diesen vgl. unten 7.3

28 Vgl. auch Huster 2014: 251ff.

29 Vgl. Althammer 2008: 289; Boldt/Schöllhorn 2008: 995ff.; Kern/Beske/Lescow 1999: 113ff.; Kuhlmann 1998:

11ff.; Schultheiss 2004: 221ff. und 568ff.

$30 \mathrm{Vgl}$. Strech et al. 2008: 1ff.; Strech/Danis/Löb/Marckmann 2009: 1261ff. 
dungsträger vorteilhaft sein, weil sie ihnen eine ausdrückliche Auseinandersetzung mit dem heiklen Thema der Leistungsbeschränkungen erspart; sie wirft aber aufgrund ihrer mangelnden Transparenz erhebliche rechtliche Bedenken auf: ${ }^{31}$

Mit rechtsstaatlichen Vorgaben entsteht insoweit ein Konflikt, als die Zuteilung medizinischer Leistungen nicht vorhersehbar ist, wenn es an ausdrücklichen Regelungen fehlt; dies nimmt dem Bürger die Möglichkeit, sich - etwa durch den Abschluss einer Zusatzversicherung - auf die Situation einzustellen; zudem laufen in derartigen intransparenten Situationen auch die Rechtsschutzmöglichkeiten praktisch leer.

Dass die Verteilungsmaßstäbe in Knappheitssituationen unklar bleiben, ist ferner unter sozialstaatlichen Gesichtspunkten bedenklich, weil nicht zu Unrecht vermutet wird, dass sich in den ungeregelten Verhandlungsprozessen, die dadurch entstehen, häufig die soziale und sprachliche Kompetenz von Mitgliedern der Ober- und Mittelschicht durchsetzen wird. ${ }^{32}$

All dies ist nicht nur für das Arzt-Patienten-Verhältnis belastend, sondern wirft schließlich auch für die demokratische Ordnung ein Problem auf: Denn entschieden wird hier über die Zuteilung wichtiger Güter letztlich von Personen - nämlich den behandelnden Ärzten -, die dazu in keiner Weise demokratisch legitimiert sind.

Eine implizite Rationierung ist daher in rechtsstaatlicher, sozialstaatlicher und demokratischer Hinsicht sehr viel bedenklicher als eine transparente Leistungsbeschränkung, etwa durch KSLL. Auch dies sollte gegenüber verfassungsrechtlichen Verfestigungen von Leistungsansprüchen und einem rigoristischen Bewertungsverbot mitberücksichtigt werden.

\subsubsection{Zwischenergebnis}

Dass Leistungseinschränkungen im öffentlichen Versorgungssystem grundsätzlich möglich sind, liegt auf der Hand. Klärungsbedürftig ist aber, von wem, in welchem Verfahren und nach welchen Kriterien sie vorgenommen werden können.

\subsection{Formelle Vorgaben}

Zunächst stellt sich die Frage, wer über den Erlass von KSLL in welchem Verfahren und auf welcher Rechtsgrundlage entscheiden soll.

31 Vgl. Francke 2003: 98; Huster et al. 2007: 703ff.

32 Vgl. Simon 1998: 81ff.; Huster 2011: 33. 


\subsubsection{Die Erforderlichkeit einer hinreichend bestimmten Rechtsgrundlage}

Denkt man innerhalb des bestehenden Systems der GKV, so wäre es zunächst nahe liegend, dass der Cemeinsame Bundesausschuss (G-BA) in seinen Richtlinien nach $\mathbb{5} 92$ SGB V die KSLL beschließt, die dann für die Leistungserbringer und die Versicherten verbindlich wären. Die Frage ist aber, ob dafür bereits eine (zudem hinreichend bestimmte) Rechtsgrundlage zur Verfügung steht.

\section{a) Kostensensible Leitlinien als „wesentliche“ Entscheidungen}

Entscheidungen über Leistungseinschränkungen in der GKV gehören - jedenfalls im Kernbereich der medizinischen Versorgung - gewiss zu den „wesentlichen“ Entscheidungen im Sinne der Wesentlichkeitstheorie des Bundesverfassungsgerichts, 33 wenn aus Kostengründen ein medizinischer Zusatznutzen verweigert wird. Auch der Erlass von KSLL unterliegt daher dem Grundsatz des Vorbehalts des Gesetzes.

\section{b) Das Wirtschaftlichkeitsgebot als Rechtsgrundlage?}

Eine ausdrückliche Grundlage für den Erlass von KSLL finden wir (bisher) im SGB V nicht. Eine entsprechende Ermächtigung könnte sich bereits aus dem Wirtschaftlichkeitsgebot des $\mathbb{1} 12$ SGB V, auf das auch $\mathbb{5} 92$ SGB V Bezug nimmt, ergeben. Allerdings ist die Bedeutung des Wirtschaftlichkeitsgebots insoweit nicht ganz geklärt:

Nach einer Interpretation bedeutet „Wirtschaftlichkeit“, dass von zwei Maßnahmen mit gleichem oder zumindest vergleichbarem medizinischen Nutzen nur die kostengünstigere zur Verfügung gestellt wird. ${ }^{34}$ Es handelt sich dann um einen reinen Kosten-Kosten-Vergleich, der normativ relativ unproblematisch ist, für die KSLL aber nicht weiterhilft, weil hier per definitionem ein medizinischer Zusatznutzen verweigert wird.

Nach einer anderen Interpretation erlaubt der Wirtschaftlichkeitsbegriff auch eine Kosten-Nutzen-Bewertung, sodass auch Maßnahmen mit geringem oder begrenzten Zusatznutzen, aber (dafür) hohen Kosten von der GKV-Versorgung ausgeschlossen werden können. Darauf könnten Ausführungen des Bundessozialgerichts in der Clopidogrel-Entscheidung hindeuten; 35 auch der G-BA nimmt in seiner Verfahrensordnung die Kompetenz für Kosten-Nutzen-Abwägungen in Anspruch. ${ }^{36}$

33 Zur Wesentlichkeitstheorie vgl. BVerfGE 33, 1 (10ff.); 34, 165 (192f.); 45, 400 (417f.); 58, 257 (268).

34 So etwa Engelhard 2007: Rn. 107ff.

35 Vgl. BSG, Urteil vom 31.05.2006, B 6 KA 13/05, Rn. 74 der Juris-Fassung.

36 Vgl. 2. Kap. \10 II VerfO G-BA. 
Die letztgenannte Auffassung ist dem Bedenken ausgesetzt, dass sie dem G-BA einen sehr weitgehenden Gestaltungsspielraum einräumt, obwohl das SGB V nicht einmal annäherungsweise zu erkennen gibt, wann denn das KostenNutzen-Verhältnis so schlecht wird, dass von der „Unwirtschaftlichkeit“ einer Maßnahme auszugehen ist. Selbst wenn man sich ihr anschließt, bleibt es allerdings fraglich, ob für den systematischen Einsatz von KSLL diese Rechtsgrundlage hinreichend bestimmt ist oder ob der Gesetzgeber nach dem Grundsatz des Parlamentsvorbehalts nicht genauere Vorgaben machen müsste. ${ }^{37}$

\subsubsection{Die Legitimation des Entscheidungsorgans}

Damit hängt ein zweites Problem zusammen. Der Vorbehalt des Gesetzes und der Parlamentsvorbehalt gehen - neben ihrer rechtsstaatlichen Wurzel - wesentlich auf den Gedanken zurück, dass die Ausübung von Staatsgewalt demokratisch legitimiert sein muss. ${ }^{38}$ Dabei beruht das Erfordernis des Vorliegens eines Gesetzes und dessen hinreichender inhaltlicher Bestimmtheit auf dem Prinzip der sachlich-inhaltlichen Legitimation der Entscheidung. Daneben muss aber auch der jeweilige Entscheidungsträger personell hinreichend legitimiert sein.

Zur Legitimation des G-BA und insgesamt der „Gemeinsamen Selbstverwaltung“ existiert bereits eine ausführliche und komplexe Diskussion, die zu keinem klaren Ergebnis geführt hat und hier nicht wiederholt werden kann. ${ }^{39}$ Nur einige Hinweise und Fragen dazu:

Sachlich-inhaltliche und personelle Legitimation stehen in Wechselwirkung miteinander: Je schwächer der eine Zweig ausgestaltet ist, desto stärker muss der andere gesichert sein. ${ }^{40}$ Angesichts der zurzeit vielleicht noch hinreichenden, aber sicherlich nicht besonders stark ausgeprägten personellen Legitimation des G-BA fällt nun die Unbestimmtheit der gesetzlichen Vorgaben besonders ins Gewicht. Dabei ist auch zu berücksichtigen, dass Entscheidungen über KSLL zwar hinsichtlich der Ermittlung des medizinischen Nutzens und der Kosten Sachverstand und wissenschaftliche Erkenntnisse voraussetzen, aber auch evidente Wertungsanteile enthalten - insbesondere hinsichtlich der noch akzeptablen Schwelle des Kosten-Nutzen-Verhältnisses. Derartige Entscheidungen sind aber originär politischer Natur und können daher auch nicht unter dem Gesichtspunkt des Sachverstandes an nur schwach legitimierte Gremien delegiert werden. ${ }^{41}$

\footnotetext{
37 Ablehnend auch Hauck 2010: $197 f$.

38 Siehe Fn. 33.

39 Vgl. nur Axer 2000: 391ff. und passim; Butzer/Kaltenborn 2001: 333ff.; Hänlein 2001: 453ff.; Hase 2005: 391ff.; Rixen 2005: 184ff.; Schmidt-Aßmann 2001: 82ff.; Schnapp 2004: 497ff.

40 Vgl. BVerfGE 107, 59 (Rn. 111); Schliesky 2004: 289.

$41 \mathrm{Vgl}$. auch Hauck 2010: 200.
} 
Jedenfalls ein systematischer Einsatz von KSLL verlangt daher, dass entweder das Gesetz deutliche Entscheidungsvorgaben benennt oder dass die politisch verantwortliche Instanz - also das Gesundheitsministerium - letztlich die Entscheidungen trifft (etwa im Wege einer Rechtsverordnung). Diese Instanzen können dabei von Sachverständigengremien und den Organen der Gemeinsamen Selbstverwaltung unterstützt werden, aber diese hätten dann nur beratende und keine entscheidende Funktion. ${ }^{42}$

\subsection{Materielle Vorgaben}

Neben der Thematik, wer in welchem Verfahren entscheidet, stellt sich die Frage, welche inhaltlichen Vorgaben diese Entscheidungen zu beachten haben. Insbesondere verfassungsrechtliche Wertungen sind insoweit zu berücksichtigen. Dabei kann es nicht grundsätzlich unzulässig sein, Kostenaspekte mit zu berücksichtigen. ${ }^{43}$ Aber welche Einschränkungen bestehen dabei? Die Probleme, die KSLL insoweit aufwerfen, entsprechen dabei vielfach denen, die Kosten-Nutzen-Bewertungen generell mit sich bringen. ${ }^{44}$

\subsubsection{Freiheitsrechtliche Probleme: Kein Raum für Kostenaspekte bei lebens- bedrohlichen oder regelmäßig tödlichen Erkrankungen?}

\section{a) Die Verfassungsrechtsprechung zu Leistungsansprüchen in der GKV}

Lange Zeit hatte das Bundesverfassungsgericht sehr zurückhaltend entschieden, dass sich aus den Grundrechten und dem Sozialstaatsprinzip kein verfassungsrechtlicher Anspruch auf Bereithaltung spezieller Gesundheitsleistungen ergibt; ${ }^{45}$ die objektivrechtliche Pflicht des Staates, sich schützend und fördernd vor die Rechtsgüter des Art. 2 Abs. 2 S. 1 GG (Leben und körperliche Unversehrtheit) zu stellen, sei unter Berücksichtigung der Gestaltungsfreiheit der zuständigen staatlichen Stellen lediglich darauf gerichtet, dass die öffentliche Gewalt Vorkehrungen zum Schutz des Crundrechts trifft, die nicht völlig ungeeignet oder unzulänglich sind. ${ }^{46}$

Eine gewisse Trendwende hat insoweit die sog. Nikolaus-Entscheidung vom 6.12.2005 eingeleitet: Danach ist es mit Art. 2 Abs. 1 GG in Verbindung mit dem Sozialstaatsprinzip und mit Art. 2 Abs. 2 GG nicht vereinbar, einen gesetzlich Krankenversicherten, für dessen lebensbedrohliche oder regelmäßig tödliche

\footnotetext{
42 Zu dieser Unterscheidung und in der Sache ebenso vgl. Schmidt-Aßmann 2004: 1692ff.

43 Vgl. oben 7.1

44 Zur Methodik der Kosten-Nutzen-Bewertung in der Gesetzlichen Krankenversicherung vgl. Dettling 2008: 379ff.; Münkler 2015; Huster 2008: 449ff.; Schaller 2009: 439ff.

45 Vgl. BVerfGE 39, 302 (314); 41, 126 (153f.); 82, 60 (80); 94, 241 (263); 115, 25 (44); BVerfG (Kammer), NJW 1997, 3085 (3085); NJW 1998, 1775 (1776).

46 Vgl. BVerfGE 77, 170 (215); 85, 191 (212); 92, 26 (46); BVerfG (Kammer), MedR 1997, 318 (318).
} 
Erkrankung eine allgemein anerkannte, medizinischem Standard entsprechende Behandlung nicht zur Verfügung steht, von der Leistung einer von ihm gewählten, ärztlich angewandten Behandlungsmethode auszuschließen, wenn eine nicht ganz entfernt liegende Aussicht auf Heilung oder auf eine spürbare positive Einwirkung auf den Krankheitsverlauf besteht. ${ }^{47}$ In einer späteren Entscheidung halten die Karlsruher Richter einen unmittelbaren grundrechtlichen Leistungsanspruch sogar dann für möglich, wenn bereits eine negative Entscheidung des G-BA vorliegt. ${ }^{48}$ In diesem Sinne hat der Gesetzgeber dann auch die Grundsätze dieser Entscheidung in das SCB V aufgenommen (vgl. $\$ 2$ Abs. 1a).

\section{b) Übertragbarkeit auf Leistungsbegrenzungen durch KSLL?}

Wenn in Fällen einer lebensbedrohlichen oder regelmäßig tödlichen Erkrankung nicht einmal Methoden mit einem - zurückhaltend formuliert - extrem unsicheren Nutzen ausgeschlossen werden können, liegt die Folgerung nahe, dass in diesen Fällen auch der Hinweis auf das schlechte Kosten-Nutzen-Verhältnis einer Maßnahme einen Leistungsausschluss nicht rechtfertigen kann.

Hinsichtlich der vom Forschungsverbund erstellten KSLL zu Medikamentefreisetzenden Koronarstents (drug eluting stents, DES) ist jedoch entscheidend, dass medikamentenbeschichtete Stents zwar durchaus zur Behandlung lebensbedrohlicher Erkrankungen eingesetzt werden, zu diesen aber mit den nicht-beschichteten Stents eine Behandlungsalternative besteht. Der Zusatznutzen der Beschichtung liegt nicht in der Verringerung der Mortalität, sondern lediglich in der Vermeidung zusätzlicher Krankenhausaufenthalte und operativer Eingriffe. Mangels Alternativlosigkeit ist somit ein verfassungsrechtlicher Anspruch auf DES nach den Crundsätzen der Nikolaus-Beschlusses ausgeschlossen.

Anders verhält es sich hingegen bei den Internen Cardioverter-Defibrillatoren (ICD). Hier sind Fälle denkbar, in denen keine Therapiealternative zur Verfügung steht und der Einsatz eines ICD zur Lebensverlängerung notwendig ist. Es ist dann fraglich, ob ein Leistungsausschluss mit den Vorgaben der Rechtsprechung vereinbar wäre. Die grundsätzliche Verbindlichkeit der KSLL, die eben nicht nur eine Handlungsempfehlung darstellt, sondern Leistungsansprüche definitiv ausschließt, müsste dann möglicherweise im eingehend zu begründenden und gegebenenfalls gerichtlich zu überprüfenden Einzelfall durchbrochen werden.

47 Vgl. BVerfGE 115, 25ff.; umfassend zur darauf folgenden Rechtsprechung Bohmeier/Penner 2009: 65ff. 48 Vgl. BVerfG (Kammer), NZS 2008, 365 (368). 


\section{c) Die Ergänzungsbedürftigkeit von Kosten-Nutzen-Erwägungen}

Die oben beschriebene Rechtsprechung des Bundesverfassungsgerichts zur Leistungspflicht der Gesetzlichen Krankenversicherung in Fällen von lebensbedrohlichen Krankheiten ist zwar sowohl hinsichtlich ihrer Begründung als auch ihres Ergebnisses äußerst umstritten. ${ }^{49}$ Sie weist aber auf ein grundsätzliches Problem des Instruments der KSLL hin: dass nämlich das Kosten-Nutzen-Verhältnis nach verbreiteter Ansicht nicht das einzige Allokationskriterium sein kann. Auch die ZEKO hat in der bereits erwähnten Stellungnahme darauf hingewiesen, dass neben dem erwarteten medizinischen Nutzen und der Kosteneffektivität einer Versorgungsleistung auch die medizinische Bedürftigkeit, verstanden als Schweregrad und Gefährlichkeit der Erkrankung und Dringlichkeit des Eingreifens, zu berücksichtigen ist. ${ }^{50}$ Dies entspricht einer weit verbreiteten Intuition, dass neben den „utilitaristischen“ Kriterien des Nutzens und der Kosteneffektivität auch die jeweilige individuelle Bedürftigkeit bei Priorisierungsentscheidungen eine Rolle spielen muss. Hier stellen sich Fragen, die juristisch noch nicht diskutiert sind:

- Geht der verfassungsrechtliche Rigorismus mit Bezug auf den „Höchstwert Leben" wirklich so weit, dass selbst Maßnahmen, die bei sehr hohen (Zusatz-)Kosten nur zu einem sehr geringen (Zusatz-)Nutzen - etwa einer geringfügigen Lebensverlängerung bei sehr schlechter Lebensqualität - führen, nicht von der Versorgung ausgeschlossen werden können?

- Inwieweit sind dringlichkeits- und bedürftigkeitsbezogene Gesichtspunkte in QALY-Bewertungen, auf denen auch die hier vorgestellten KSLL beruhen, bereits hinreichend integriert?

\section{d) Das ungeklärte Verhältnis von Gerechtigkeits- und Effizienzüberlegungen}

Der Hintergrund dieser Fragen besteht darin, dass die Kombination von nutzen- und bedürftigkeitsbezogenen Gesichtspunkten erhebliche Folgeprobleme aufwirft, weil bis jetzt keine anerkannte Theorie existiert, wie die unterschiedlichen Aspekte zu gewichten sind: Die Bedürftigkeit (also eine originär gerechtigkeitsbezogene Überlegung) kann Kosten- und Nutzen-Erwägungen (also Effizienzerwägungen) aus dem Feld schlagen. Aber es ist unklar, wann und inwieweit dies geschehen muss und soll. Das Problem wird sich auch nicht so einfach lösen lassen, weil es die Crundlagen unseres normativen Haushalts betrifft: Wie lässt sich die utilitaristische Orientierung der Kriterien des Nutzens und der Kosteneffektivität damit vereinbaren, dass wir im Übrigen den Utilitarismus strikt ablehnen und uns an Gleichheit, Gerechtigkeit und individuellen Rechten orientieren? ${ }^{51}$

49 Zur Kritik vgl. Heinig 2006: 771ff.; Huster 2006: 466ff.; Ulmer 2007: 585ff.; Wenner 2009: 178.

$50 \mathrm{Vgl.} \mathrm{Fn.} 23$.

51 Vgl. dazu nur Lübbe 2001: 149f. 


\subsubsection{Gleichheitsrechtliche Probleme: Unzulässige Diskriminierung bestimmter Subgruppen?}

\section{a) Die gleichheitsrechtliche Dimension}

Neben den originären verfassungsrechtlichen Ansprüchen, die aus den Freiheits- als Leistungsrechten abgleitet werden können, ist es auch möglich, dass KSLL auf Differenzierungskriterien beruhen, die in Konflikt mit den Gleichheitsrechten geraten.

\section{b) Rechtfertigung über Ex ante-Interessen}

KSLL führen dazu, dass bestimmten Patientensubgruppen ein medizinischer Zusatznutzen aufgrund eines schlechten Kosten-Nutzen-Verhältnisses verweigert wird. Dies kann dann gerechtfertigt sein, wenn jeder in die Situation dieser Subgruppe kommen kann, weil man dann unterstellen kann, dass die Differenzierung auf unparteiischen Ex ante-Interessen beruht. Sehr viel heikler ist es dagegen, wenn dieses Risiko nicht zufällig streut, sondern Cruppen trifft, die sich vorher bestimmen lassen, weil dann die Gefahr der Diskriminierung dieser Gruppen droht. ${ }^{52}$

Wenn z.B. Menschen mit einer Behinderung im Rahmen von QALY-Berechnungen systematisch benachteiligt werden, könnte dies sogar ein Problem des Art. 3 Abs. 3 S. 2 GG darstellen. Aber auch bei der Benachteiligung von Personen mit chronischen „teuren“ Krankheiten ist zu überlegen, ob derartige Differenzierungen mit dem allgemeinen Gleichheitssatz vereinbar sind. Die Krankheit stellt nämlich ein personenbezogenes Merkmal dar, das sich im Rahmen der „Neuen Formel“ des Art. 3 Abs. 1 GG einer besonders strengen Überprüfung stellen müsste. ${ }^{53}$

Für die KSLL zu ICD und DES dürften insoweit aber keine Probleme bestehen, weil die ausgeschlossenen Subgruppen jeweils nicht von vornherein bestimmbar sind. Sie werfen daher keine besonderen gleichheitsrechtlichen Fragen auf.

\subsection{Fazit}

1. Kosten-Nutzen-Erwägungen sind bei Allokationsentscheidungen nicht grundsätzlich ausgeschlossen. Auch KSLL sind daher in materiellrechtlicher Hinsicht ein grundsätzlich zulässiges Steuerungsinstrument. Wenn man hier nicht einen unplausiblen verfassungsrechtlichen Rigorismus vertreten will und keine von vornherein identifizierbare Subgruppe betroffen ist, sind KSLL zulässig.

52 Vgl. dazu auch Huster 2012: $567 f$.

53 Vgl. Bieback 2006: 83f.; Bouchouaf 2006: 315; Huster 2005: Rn. 96; Welti 2008: 62. 
2. Ihr Einsatz bedarf allerdings einer hinreichend bestimmten Rechtsgrundlage und eines ausreichend legitimierten Entscheidungsträgers. Hier stellen sich schwierige Fragen der Aufteilung der Entscheidungskompetenzen zwischen Gemeinsamer Selbstverwaltung und Gesundheitspolitik.

\section{Literatur}

Althammer I (2008) Rationierung im Gesundheitswesen aus ökonomischer Perspektive, Sozialer Fortschritt, 289-294

Augurzky B, Tauchmann H, Werblow A, Felder S (2009) Effizienzreserven im Gesundheitswesen. RWI: Materialien Heft 49: Essen

Axer P (2000) Normsetzung der Exekutive in der Sozialversicherung. Mohr Siebeck: Tübingen

Bieback K-I (2006) Altersdiskriminierung: Grundsätzliche Strukturen und sozialrechtliche Probleme, Zeitschrift für Sozialreform, 75-99

Bohmeier A, Penner A (2009) Die Umsetzung des Nikolaus-Beschlusses durch die Sozialgerichtsbarkeit: Fortentwicklung und Widersprüche zu den Vorgaben des BVerfG, Wege zur Sozialversicherung, 65-85

Boldt I, Schöllhorn T (2008) Intensivmedizinische Versorgung: Rationierung ist längst Realität, Deutsches Ärzteblatt, 995-997

Bosbach G, Bingler K (2008) Droht eine Kostenlawine im Gesundheitswesen?, Soziale Sicherheit, 5-12

Bouchouaf S (2006) Statistische Altersdiskriminierung, Kritische Justiz, 310-319

Braun B, Kühn H, Reiners H (1998) Das Märchen von der Kostenexplosion - populäre Irrtümer zur Gesundheitspolitik, Fischer Taschenbuch Verlag: Frankfurt am Main

Butzer H, Kaltenborn M (2001) Die demokratische Legitimation des Bundesausschusses der Ärzte und Krankenkassen, Medizinrecht, 333-342

Dabrock P (2005) Befähigungsgerechtigkeit als Kriterium zur Beurteilung von Grundversorgungsmodellen im Gesundheitswesen. In: Rauprich 0, Marckmann G, Vollmann I (Hrsg.) Gleichheit und Gerechtigkeit in der modernen Medizin. 213-247. Mentis-Verlag: Paderborn

Deppe U (1998). Neoliberalismus und Gesundheitspolitik in Deutschland - Menschenrechte lassen sich nicht kommerzialisieren, ohne daß sie daran zerbrechen. In: Kaupen-Haas H, Rothmaler C (Hrsg), Strategien der Gesundheitsökonomie. 101-114. Mabuse-Verlag, Frankfurt am Main

Dettling H-U (2008) Die Kosten-Nutzen-Bewertung bei Arzneimitteln im Schnittfeld von Ökonomie und Recht, Vierteljahresschrift für Sozialrecht, 379-399

Ebsen I (1997) Ressourcenknappheit im Gesundheitswesen - verfassungsrechtliche Implikationen. In: Ratajczak T, Bergmann KO (Hrsg.) Die Budgetierung des Gesundheitswesens. 109-127. Springer-Verlag: Berlin Heidelberg

Francke R (2003) Begrenzung der Leistungen der gesetzlichen Krankenversicherung - Grund- und Wahlleistungen, Rationierung, Priorisierung, Gesundheitsrecht, 97-101

Engelhard W (2007) $\$ 12$. In: Schlegel R, Engelmann K (Hrsg.) juris-PK SGB V, Stand der Online-Version 1.8.2007

Glaeske G (2007) Effizienzoptimierung in der Arzneimittelversorgung - Für mehr Rationalität und Wirtschaftlichkeit in der Therapie mit Medikamenten. In: Ulrich V, Ried W (Hrsg.) Effizienz, Qualität und Nachhaltigkeit im Gesundheitswesen, Festschrift für Wille. 553-564. Nomos: Baden-Baden

Hänlein A (2001) Rechtsquellen im Sozialversicherungsrecht. Springer Verlag: Berlin Heidelberg

Hase F (2005) Verfassungsrechtliche Bewertung der Normsetzung durch den Gemeinsamen Bundesausschuss. Medizinrecht, 391-397

Hauck E (2010) Kostensensible Leitlinien als Rationierungsinstrumente in der GKV?. Die Sozialgerichtsbarkeit, 193200

Heinig HM (2006) Hüter der Wohltaten?, Neue Zeitschrift für Verwaltungsrecht, 771-774 
Heiß H (2003) Rationierung aus ärztlicher Sicht. In: Marckmann G (Hrsg.) Gesundheitsversorgung im Alter. 123-129. Schattauer: Stuttgart

Held C (2011) Altersgrenzen als zulässiges Rationierungskriterium in der Gesetzlichen Krankenversicherung?. Dr. Kovač: Hamburg

Höfling W, Augsberg S (2009), Leistungen der gesetzlichen Krankenversicherung unter Finanzierungsvorbehalt?. Zeitschrift für medizinische Ethik, 45-60

Höfling W (1998) Rationierung von Gesundheitsleistungen im grundrechtsgeprägten Sozialstaat. In: Feuerstein G, Kuhlmann E (Hrsg.) Rationierung im Gesundheitswesen. 143-155. Ullstein Medical: Wiesbaden

Hoppe I D (2008) Rationierungszwang und Ressourcen - Allokation in einer älter werdenden Gesellschaft. Sozialer Fortschritt, 304-306

Huster S (2005) Art. 3. In: Friauf K-H, Höfling W (Hrsg.) Berliner Kommentar zum Grundgesetz. Erich Schmidt Verlag: Berlin

Huster S (2006) Anmerkung zu BVerfG v. 6.12.2005 - 1 BvR 347/98. Juristenzeitung, 466-468

Huster S (2008) Die Methodik der Kosten-Nutzen-Bewertung in der Gesetzlichen Krankenversicherung. Gesundheitsrecht, 449-458

Huster S (2010) Knappheit und Verteilungsgerechtigkeit im Gesundheitswesen. Deutsches Verwaltungsblatt, 1069-1077

Huster S (2011) Soziale Gesundheitsgerechtigkeit. Wagenbach: Berlin

Huster S (2012) Vulnerable Patientengruppen und Leistungsbeschränkungen im öffentlichen Versorgungssystem, Medizinrecht, 565-570

Huster S (2014) Hat das Leben keinen Preis? Absolute und relative Ansprüche im System der Gesundheitsversorgung. In: Hruschka J, Joerden IC (Hrsg.) Jahrbuch für Recht und Ethik/Annual Review of Law and Ethics 22. 251-267. Duncker \& Humblot: Berlin

Huster S, Strech D, Marckmann G, Freyer D, Börchers K, Neumann A, Wasem I, Held C (2007) Implizite Rationierung als Rechtsproblem. Ergebnisse einer qualitativen Interviewstudie zur Situation in deutschen Krankenhäusern. Medizinrecht, 703-706

Huster S, Kliemt H (2009) Opportunitätskosten und Jurisprudenz. Archiv für Rechts- und Sozialphilosophie 95, $241-251$

Jaeger R (2003) Die Reformen in der Gesetzlichen Sozialversicherung im Spiegel der Rechtsprechung. Neue Zeitschrift für Sozialrecht, 225-234

Kern A, Beske F, Lescow H (1999) Auswertung einer Leserumfrage: Leistungseinschränkung oder Rationierung im Gesundheitswesen? Deutsches Ärzteblatt, 113-117

Kingreen T (2008) Gesundheit ohne Gesetzgeber?. In: Kingreen T, Laux B (Hrsg.) Gesundheit und Medizin im interdisziplinären Diskurs. 150-176. Springer Verlag: Berlin Heidelberg

Kliemt H (2006) Ethische Aspekte der Gesundheitsversorgung bei Ressourcenknappheit. In: Brink A, Eurich I, Hädrich I, Langer A, Schröder P (Hrsg.) Gerechtigkeit im Gesundheitswesen. 45-61. Duncker \& Humblot: Berlin

Kollwitz AA (1999) Verteilungsgerechtigkeit und Generationenkonflikt. Berliner Medizinethische Schriften Heft 35. Humanitas-Verlag: Dortmund

Kopetsch T (2000) Zur Rationierung medizinischer Leistungen im Rahmen der Gesetzlichen Krankenversicherung. Nomos Verlag: Baden Baden

Krämer W (1996) Medizin muss rationiert werden. Medizinrecht, 1-5

Krause P (1982) Eigentum an subjektiven öffentlichen Rechten. Duncker \& Humblot: Berlin

Kühn H (2005) Demografischer Wandel und GKV - Kein Grund zur Panik. Die Krankenversicherung, 178-183

Kuhlmann E (1998) „Zwischen zwei Mahlsteinen“ - Ergebnisse einer empirischen Studie zur Verteilung knapper medizinischer Ressourcen in ausgewählten klinischen Settings. In: Feuerstein G, Kuhlmann E (Hrsg.) Rationierung im Gesundheitswesen. 11-80. Ullstein Medical: Wiesbaden

Lübbe W (2001) Veralltäglichung der Triage?, Überlegungen zu Ausmaß und Grenzen der Opportunitätskostenorientierung in der Katastrophenmedizin und ihrer Übertragbarkeit auf die Alltagsmedizin. Ethik in der Medizin, $148-160$ 
Marckmann G (2005) Rationalisierung und Rationierung: Allokation im Gesundheitswesen zwischen Effizienz und Gerechtigkeit. In: Kick A, Taupitz I (Hrsg.) Gesundheitswesen zwischen Wirtschaftlichkeit und Menschlichkeit. 179-201. LIT: Münster

Marckmann G (2008) Gesundheit und Gerechtigkeit. Bundesgesundheitsbl - Gesundheitsforsch - Gesundheitsschutz, 887-894

von Maydell B (2004) Rechtsstaatliche Voraussetzungen einer Rationierung von Gesundheitsleistungen. In: Albrecht P, Lorenz E, Rudolph B (Hrsg.) Risikoforschung und Versicherung. Festschrift für Helten. 353-367. VVW: Karlsruhe

von Maydell B (2005) Rationierung in unterschiedlichen Gesundheitssystemen. ArztRecht, 88-108

Münkler L (2015) Kosten-Nutzen-Bewertungen in der gesetzlichen Krankenversicherung. Duncker \& Humblot: Berlin

Oberender P (1998) Gesundheitsversorgung zwischen ökonomischer und medizinischer Orientierung. In: Nagel E, Fuchs C (Hrsg.), Rationalisierung und Rationierung im deutschen Gesundheitswesen. 10-26. Thieme: Stuttgart New York

Reiners H (2009) Mythen der Gesundheitspolitik. Hans Huber: Bern

Rixen S (2005) Sozialrecht als öffentliches Wirtschaftsrecht. Mohr Siebeck: Tübingen

Schaller BI (2009) Funktionsweise und Probleme der Kosteneffektivitätsanalyse im Gesundheitswesen. Das Krankenhaus, 439-445

Schlegel R (2004) Übergangsrecht - Aspekte von Rückwirkung und Vertrauensschutz, Vierteljahresschrift für Sozialrecht, 313-347

Schliesky U (2004) Souveränität und Legitimität von Herrschaftsgewalt. Mohr Siebeck: Tübingen

Schmidt-Aßmann E (2001) Grundrechtspositionen und Legitimationsfragen im öffentlichen Gesundheitswesen. Walter de Gruyter: Berlin/New York

Schmidt-Aßmann E (2004) Verfassungsfragen der Gesundheitsreform, Neue Juristische Wochenschrift, 1689-1695 Schnapp FE (2004) Untergesetzliche Rechtsquellen im Vertragsarztrecht - am Beispiel der Richtlinien. In: von Wulffen M, Krasney 0 E (Hrsg.) Festschrift 50 lahre Bundessozialgericht. 497-515. Heymanns: Köln

Schröder H, Nink K (2007) Wirtschaftliche Arzneimittelversorgung. Die Krankenversicherung, 305-308

Schultheiss C (2004) Im Räderwerk impliziter Rationierung - Auswirkungen der Kostendämpfung im deutschen Gesundheitswesen. Psychneuro, 221-228 und 568-575

Simon M (1998) Krankenhausfinanzierung und Rationierung. Zum Zusammenhang von ökonomischen Anreizen und Rationierung medizinischer Leistungen im Krankenhaus. In: Feuerstein E, Kuhlmann E (Hrsg.) Rationierung im Gesundheitswesen. 81-96. Ullstein Medical: Wiesbaden

Strech D, Börchers K, Freyer D, Neumann A, Wasem I, Marckmann G (2008) Ärztliches Handeln bei Mittelknappheit. Ergebnisse einer qualitativen Interviewstudie. Ethik in der Medizin, 94-109

Strech D, Danis M, Löb M, Marckmann G (2009) Ausmaß und Auswirkungen von Rationierung in deutschen Krankenhäusern. Deutsche Medizinische Wochenschrift, 1261-1266

Ulmer M (2007) Neue Untersuchungs- und Behandlungsmethoden. Die Sozialgerichtsbarkeit, 585-592

Vogt A (2004) Krankheitsbegriff und GKV-Mittelverteilung: Sieben Thesen aus Krankenkassen-Sicht. In: Mazouz N, Werner MH, Wiesing U (Hrsg.) Krankheitsbegriff und Mittelverteilung. 187-192. Nomos Verlag: Baden Baden

Wagner W (2009) Drohkulisse Priorisierung. Dr. med. Mabuse 4/2009, 6-14

Welti F (2008) Schutz vor Benachteiligungen im deutschen Sozialrecht nach den europäischen Gleichbehandlungsrichtlinien und ihrer Umsetzung. Vierteljahresschrift für Sozialrecht, 55-81

Wenner U (2009) Rationierung, Priorisierung, Budgetierung: verfassungsrechtliche Vorgaben für die Begrenzung und Steuerung von Leistungen der Gesundheitsversorgung, Gesundheitsrecht, 169-181

Zentrale Kommission zur Wahrung ethischer Grundsätze in der Medizin und ihren Grenzgebieten (Zentrale Ethikkommission) bei der Bundesärztekammer (2007) Stellungnahme zur Priorisierung medizinischer Leistungen im System der Gesetzlichen Krankenversicherung. URL: http://www.zentrale-ethikkommission.de/ downloads/LangfassungPriorisierung.pdf (Zugriff am 21.04.2015) 